appeared to be a carcinoma of the epididymis. It was impossible to visually or palpably demonstrate the variety of the bowel; an extreme hyperplasia of the serous and muscular coats destroyed all semblance of normal intestine. The bowel and interior of the sac were covered with a muco-purulent material of offensive odor. This secretion was wiped away and the upper portion of the bewel was protected with gauze pads. After partial separation of the adhesions, the large and small bowels were differentiated. Adhesions were broken down in the course of which a portion of the hypertrophied serous coat was stripped from the lower portion of the cecum; this demonstrated the vermiform appendix passing downward into the tumor. The appendix was ligated and cut as close to the cecum as possible. During the manipulations there was an escape of a small amount of very fetid pus from the lower part of the appendix; because of this, and because of the agglutina tion of the appendix, testicle and hydrocele and hernial sacs, it was decided to remove all en masse. All adhesions of bowels up to the internal ring were broken down and tied off and a large amount of much thickened omentum cut away at this point, and after a careful toilet, the intestines were replaced in the abdominal cavity. The spermatic cord was ligated and cut; the hypertrophied hernial sac was eut away flush with the internal abdominal ring, and removed, together with the appendix, testicle and hydrocele sac. The internal ring was closed with interrupted sutures of catgut, and the internal oblique and transversalis muscles and conjoined tendon sutured to Poupart's ligament well down to the pubic spine. United the separated external oblique aponeurosis, obliterating the external ring, and closed the skin with silkworm gut, inserted silkworm gut drainage in the upper and central portions of the skin wound, and packed the scrotum with iodoform gauze. On account of the exhausted condition of the patient, did not operate on small hernia on left side.

Patient was given 1000 c.c. of normal salt solution subcutan. eously on the table. He returned from the operating room in a feeble condition; temperature 100.2 ; pulse 106 , intermittent; respirations 44 . Sulphate of strychnia, gr. 1/30, was given hypodermically every two hours, and one pint of normal salt solution at a temperature of 115 per rectum, every three hours. He had fair bowel movement; was very restless during the night.

August 9: In much pain, vomiting and hiccoughing severely. Abdomen distended and tender; slight bowel movement; some flatus passed; average temperature 100.2; pulse 96 ; respirations 34 .

August 10: Normal salt enemas were discontinued; there was slight bowel movement. 'Temperature 100 ; pulse 85 ; respirations 36 ; severe pain, with great abdominal distension; much weakness.

August 1l: Hot turpentine stupes were kept constantly on the abdomen; there was some vomiting and hiccough. He passed a little flatus, temperature not running above 90. Predigested beef $\overline{3}$ ss was given every three hours.

August 12: General condition improved. There was no return from enemas; vomiting and hiccough were lessened.

August 13: There was slight bowel movement, with escape of a great deal of flatus. Abdominal distension was much reduced. Dressed wound, removed gauze and silkworm gut drains. Wound suppurated superficially; was repacked with iodoform gauze.

August 14: Vomiting and hiccough disappeared. There were four free bowel movements: no abdominal distension or pain; hot turpentine stupes discontinued. General condition was much improved; predigested beef continued. Temperature averaged 99; wound discharged freely at upper and lower angles.

Second week: Wound was irrigated and dressed daily, still suppurating and discharging freely. Drainage was inserted beneath skin from the upper angle to the center of the wound, and from the center to the lower angle. General condition was good, temperature averaging normal.

Third and fourth weeks: Wound still suppurated in lesser degree; sinuses beneath skin gradually closed; daily dressings, and through-and-through irrigations were continued. Bowels moved freely.
Fifth week: Temperature was normal or slightly subnor. mal; wound still discharged, but nearly closed. Bowels were in good condition.

Patient left hospital at the end of the seventh week; walking about; wound closed except very small superficial sinus, with no sign of return of hernia. Two weeks later patient reported wound entirely closed, with no return of hernia. He is doing daily work without a truss or any form of supporter.

The specimen was submitted to Dr. W. A. Evans for examination, who reported as follows: "Gross appearance: The specimen consists of a testicle with much thickened tunics and hydrocele and peritoneal sacs. The spermatic cord is very hard and tortuous, winding over surface of sacs (hydrocele and hernia). The exceedingly thick peritoneal sac contains an appendix which in its upper portion has its submucosa and mucosa stripped from the muscular tunics. The lower part has an enormously developed muscular tunic, and is embedded in a mass of old scar tissue. There is also some omentum greatly thickened. Sections were made of appendix at tip, and proximal end of the intestine. Microscopically, the appendix shows hyperpiasia in the mucosa, submucosa, muscle tunics, and in the outside fibrous coat. There is an acute inflammation engrafted upon a chronic one. The vas deferens shows an enormous hyperplasia of fibrous tissue. There is no evidence of malignant disease."

The hernia evidently had been unreduced for a long period of years. 'The adhesions of the appendix to the lower portion of the sac and testicle, and numerous bowel adhesions resulting from an extension of appendical inflammation necessitated an intense peristaltic action in the misplaced bowel, with great hypertrophy as the result.

\section{A NEW METHOD FOR THE REMOVAL OF INTERNAL HEMORRHOIDS UNDER LOCAL ANESTHESIA.}

THOS. CHAS. MARTIN, M.D.

Irresident of the American Proctologic Society, Proctologist to the Cleveland General Hospital, Professor of Proctology in the Cleveland College of P'hysicians and Surgeons. CLEVELANIS, OHIO.

The subject of hemorrhoids often prefers to bear those ills he has than fly to that relatively desperate refuge afforded by general anesthesia and be relieved. As a consequence he may become a crank or an invalid. He receives little sympathy and less help, often.

The non-malignant anal growths may be removed painlessly without resort to general anesthesia by means of the technic presently to be described, provided it be performed by the trained hands of an operator who thoroughly understands the principles of infiltration-anesthesia, and who, furthermore, has been sufficiently persevering to master the difficulties encountered in the application of these principles to this operation.

The clamp, Fig. 1. consists of a hollow cone three and one-quarter inches in length and three-quarters of an inch in diameter at its distal extremity, and one and three-yuarter inche: in diameter at its proximal end. One quadrant of the cone is fenestrated, Fig. 3. This is occupied by a movable blade with a serrated edge. which makes contact with the cone's serrated edge. The movable blade is sheathed in the cone when the jaws of the clamp are separated. Hence, after the instrument is introduced it may be made to receive the pile without irregularly expanding the anus. The great essential to painless manipulation of the sphincter is the even distribution of pressure throughout its circumference. Users of irregularly dilating speculums have been confused and baffled by the pain they provoke.

Preparation of the patient consists in daily gradual dilatation of the anus by means of Kelly's conic dilator until the anus may be made to open painlessly to the 
degree necessary for the introduction of the clamp. Those patients who long have been subjects of hemorrhoids have a dilated or relaxed sphincter, and therefore do not require this preparatory. treatment.

The patient should be placed and made comfortable in Sims' posture. The table's upright knee-piece should be adjusted to support the patient's legs, and the light focused on the field of operation.

The anoscope should now be employed that the precise situation of each hemorrhoid may be determined. These should be noted as occupying certain given zones and quadrants of the anus.

The summit of each tumor should be infiltrated with a one-tenth of one per cent. solution of eucain. A very fine needle should be employed. The needle should have a long tapering point; its introduction provokes little or no pain, and the thin wall of a varicose hemorrhoid may be infiltrated. Whereas, on the other hand, a needle of larger diameter will enter the tissues less easily and
The operator should take the well-lubricated clamp, adjusted as shown in Fig. 2, and introduce it into the anus with its blade pressing against the tumor which first is to be removed. When the instrument is buried to its shoulder the fenestrum should be opened, as shown in Fig. 3. The hemostat, which is attached to the tumor within the now exposed field, should be used as a lever to drag the hemorrhoid into the clamp's chamber. The pressure incident to the introduction of the clamp often expresses the eucain from the tumor so that it is necessary to re-anesthetize it before subjecting it to such manipulation as is necessary to carry it completely into the clamp. As the pressure of the clamp from this time on obstructs the local circulation, the infiltration should be thorough. Not only must the tumor itself be anesthetized, but its base also, and the tissues underlying this. If the patient should feel pain at any time during these manipulations, it indicates that the infiltration is incomplete-complete it. The clamp

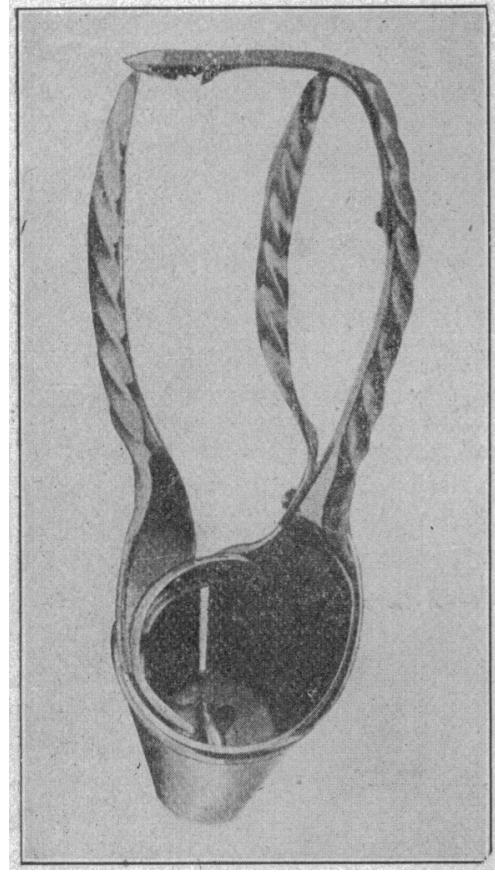

Figure 1.

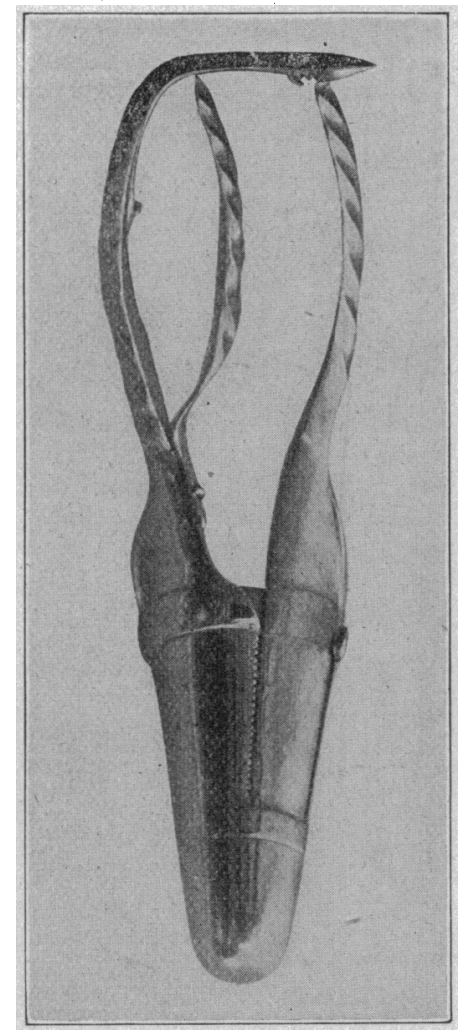

Figure 2

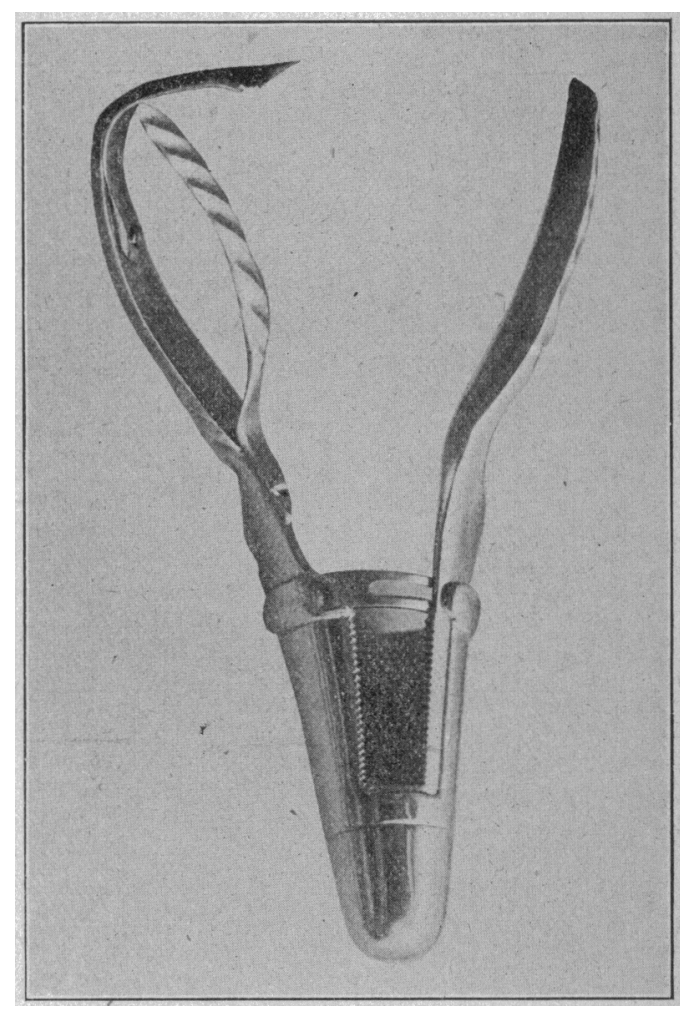

Figure 3. will provoke great pain, and perhaps may be so much thicker than the capsule of the tumor it is designed to anesthetize, that instead of effecting an infiltration of that structure the anesthetic may be driven at once into a blood-space and directly into the circulation.

The now artificially edematous and anesthetized portions of the several tumors should be grasped with curved hemostats and the anoscope withdrawn. It will then be noticed that the tumors are somewhat prolapsed under the weight of the pendant hemostats, and that because of their handles the anoscope can not be drawn off them. They should be detached, withdrawn from the anoscope and reattached, one by one. Thus, in order, each of the prolapsed tumors is to be released and again seized and the anoscope removed from field of operation.

The attached hemostats should be surrendered to an assistant, who should radiate them from the anus and well out of the ray of the operator. should now be closed and locked, and the growth cut away by means of scissors.

If the character of the growth is such that the operator has reason to fear secondary hemorrhage, the wound should be lock-stitched with catgut. If it be of the connective-tissue or fibrous variety the pedicle should be cauterized. The red-hot platinum should be applied for a second only and repeatedly, thus the clamp will not accumulate sufficient heat to distress the patient, and the lymph will be coagulated and plug the vessels.

Tentatively, the clamp should be made to release the wound. If there be any bleeding it may be controlled at once by suture or cauterization.

The clamp should be withdrawn gently and reintroduced for the removal of other hemorrhoids, if necessary.

This clamp may be separated and rendered asepticFigs. 4 and 5. As the tumor operated on is within a sterile metal chamber, the former may be antisepticized, 
and, therefore, the field of operation may be rendered surgically correct. Insufflation of iodoform or other antiseptic powder upon the wound fortifies it in a measure against subsequent infection.

Because of its peculiar form this clamp effectually

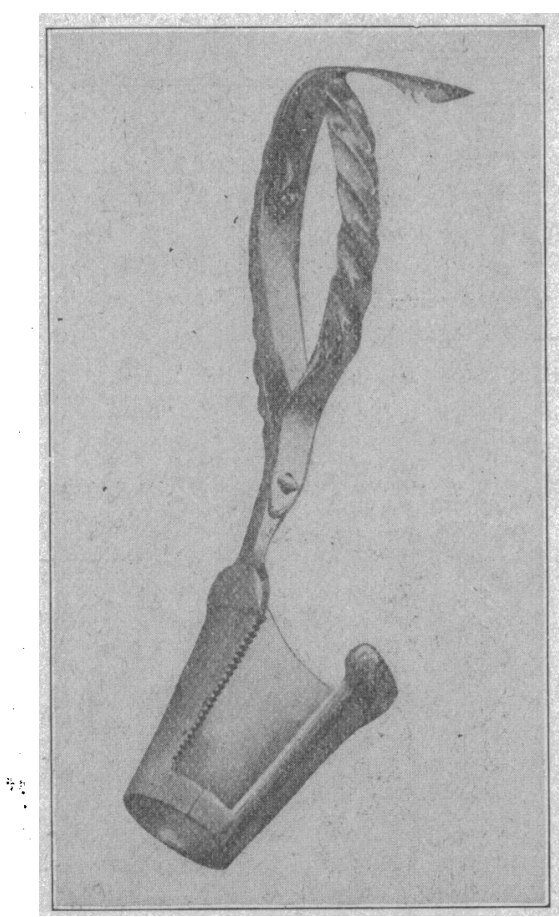

Figure 4.

blocks the field of operation against the accidental invasion of feces or the remnant of an enema. There can occur no hemorrhage to inundate and obscure the field. The pile is dry-docked.

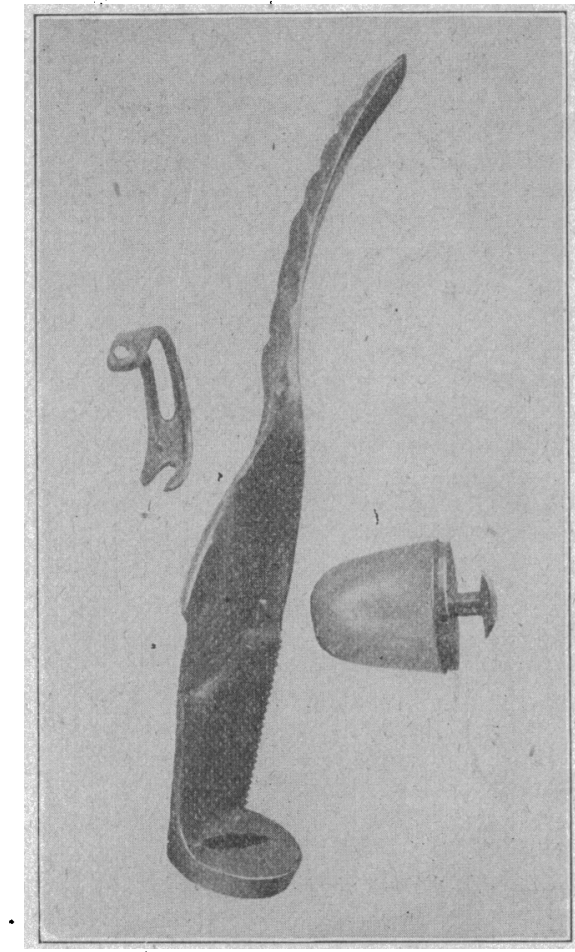

Figule $\overline{5}$.

This clamp demands that the wound shall be linear in form and parallel with the axis of the anus; it dilates the anus to a degree greater than its normal excursion, -hence there will be no subsequent contraction and stricture as a result of this operation.

That this method of clamp operation is inapplicable to inflamed or thrombotic piles, is obvious.

Eucain is much less toxic than cocain. Eucain may be sterilized by boiling without affecting its anesthetic property. An ounce or more of a one-tenth of one per cent. solution may be used without danger, for on the removal of the tumor most of the eucain is recovered'. If the eucain be prepared in normal salt solution its injection is not so likely to be followed by smarting and burning.

Local anesthesia is a surgical refinement; skill ir effecting it may be acquired only by the exercise of patience and practice.

729 Case Avenue.

\section{A METHOD OF SUTURING THE GALL-BLAD- DER TO THE PARIETES IN GALL BLADDER OPERATIONS.}

WILLJAM WOTKYNS SEYMOUR, A.B. Yale, M.D. Harvard. SURGEON TO THE SAMARITAN IIOSPITAL TROY, N. $\mathbf{Y}$.

In my last twenty cases $I$ have attaehed the gallbladder to the parietes in the following manner: A

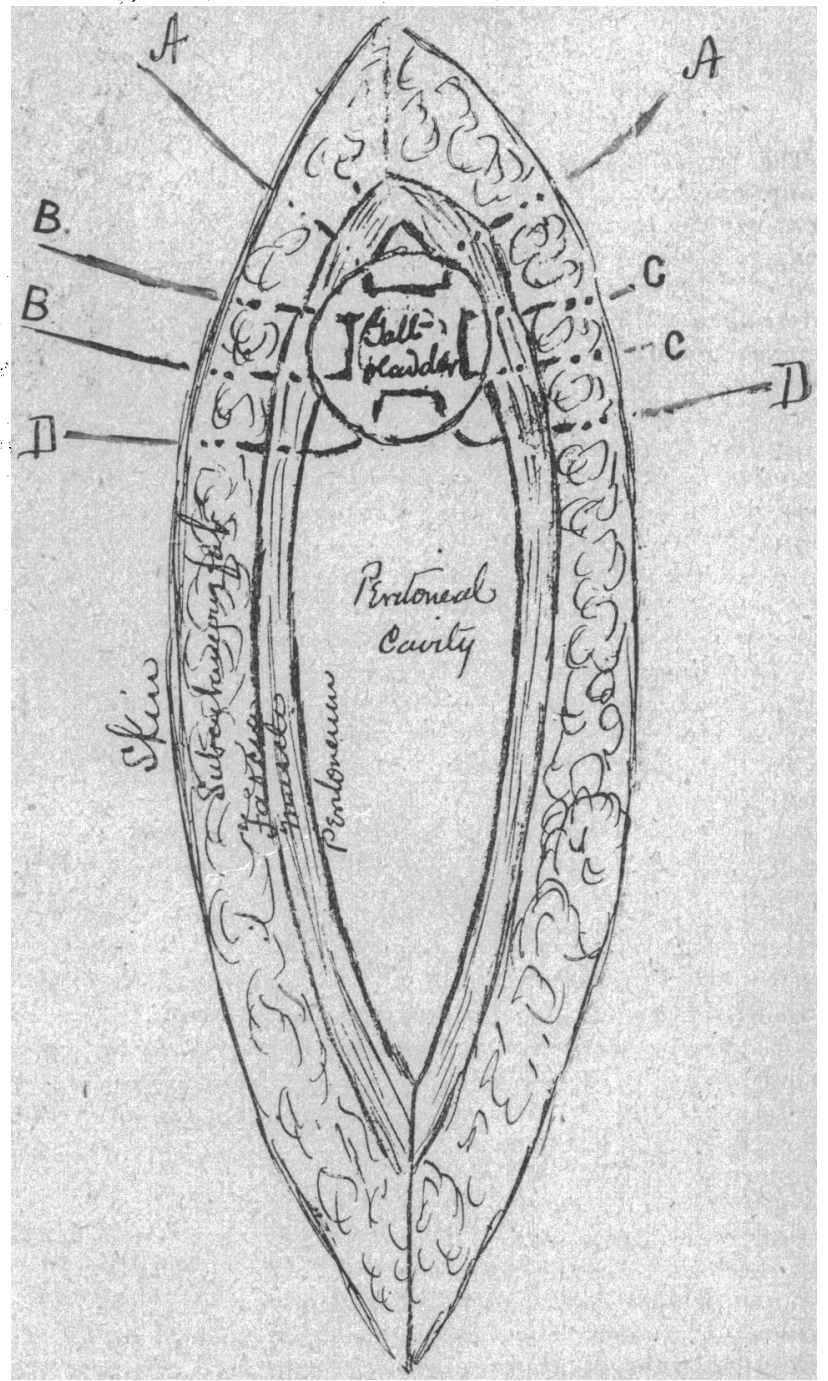

full curved Hagedorn needle is threaded with silkworm gut and passed mattress-stitch fashion from within the gall-bladder through the peritoneum: overlying muscle 